\title{
Dimensiones comunitarias. Vivir con autonomía personal: prevención e intervención en situaciones de exclusión
}

\author{
Communitary dimensions. To live with personal \\ autonomy: prevention and intervention in social \\ exclusion
}

\author{
Manuel MUÑOZ LÓPEZ* \\ SOnia PANADERO HERRERO \\ Eloísa PÉREZ SANTOS
}

\begin{abstract}
RESUMEN
Parece evidente la necesidad de contar con herramientas de clasificación y valoración precisas, que ayuden a abordar la situación de dependencia. Esta comunicación aborda principalmente el tema de la evaluación en dependencia destacando los problemas y los avances realizados en los últimos años en este sentido, teniendo en cuenta fundamentalmente la situación española. Además de abordar la importancia de la evaluación a la hora de determinar la existencia y el nivel de dependencia, se expone la evaluación de la intervención con un colectivo en riesgo real de llegar a situaciones de dependencia: las personas sin hogar con enfermedad mental grave y crónica.
\end{abstract}

\section{PALABRAS CLAVE}

Evaluación, dependencia, Funcionamiento, Autonomía, Enfermedad mental grave y crónica.

\footnotetext{
* Facultad de Psicología. Universidad Complutense de Madrid.
} 
Dimensiones comunitarias. Vivir con autonomía personal: prevención e intervención en situaciones de exclusión

\section{ABSTRACT}

Instruments to classify and assess are necessary to understand the dependence situation. This communication deals with assessment in dependence, overall the problems and advances in the last years in Spain. Also an evaluation of an intervention for a group in risk of dependence (people with severe and chronic mental illness) is discussed.

\section{KEY WORDS}

Assessment, Dependence, Functioning, autonomy, Disability, severe and chronic mental illness. 
La definición clara de conceptos y la existencia y acuerdo sobre una escala 0 método de evaluación eficaz para abordar y cuantificar el problema es un requisito necesario previo a la planificación de la atención, el estudio, etc. de cualquier fenómeno sanitario, psicológico o social. Por tanto, la evaluación es una herramienta fundamental para la planificación y estudio, entre otros fenómenos, de la dependencia, que es lo que hoy nos ocupa. Es decir, es necesario contar con herramientas de clasificación y valoración más precisas, que ayuden a abordar la situación de dependencia en la que se encuentran miles de personas en nuestro país.

Sin embargo, tras reconocer la necesidad de contar con esos métodos de valoración y graduación de la dependencia, también es importante señalar que el acuerdo de estos métodos presenta múltiples problemas. Entre otros, la consideración de diferentes áreas implica la necesidad de que participen múltiples profesionales, no sólo en valoración sino también en la definición de conceptos, etc., lo que dificulta la valoración ya que cada disciplina pone el énfasis en diferentes aspectos.

\section{Instrumentos de valoración de la dependencia}

El primer paso en la búsqueda de un método o procedimiento de evaluación de la dependencia es el establecimiento de los "requisitos" que deben cumplir. Querejeta (2003) señala los siguientes:

1. Información y consentimiento de la persona o de su tutor, en el caso de que ésta no sea capaz.

2. Aplicación universal, es decir, debe permitir su aplicación a cualquier persona, con diferentes estados de salud, etiologia y severidad de sus problemas, edad, cultura, etc.

3. Elección de las actividades a valorar según el área de dependencia a abordar (actividades de autocuidado, movilidad dentro o fuera del domicilio, etc.)

4. Claridad y concreción de las definiciones, con lenguaje sencillo, evitando ambigüedades o repeticiones, aceptado internacionalmente y que pueda ser aplicado por diferentes tipos de profesionales.

5. Debe diferenciar la necesidad de ayudas o apoyos humanos, es decir, de otra persona, de la necesidad de ayudas o apoyos materiales o técnicos.

6. Determinación del contexto en el que se desarrollan las actividades a valorar: contexto real, contexto neutral 0 estandarizado y contexto subjetivo.

7. Posibilidad de análisis desde distintos niveles de complejidad, tanto a nivel cuantitativo, como cualitativo.

Como se recoge en el Libro Blanco sobre la atención a las personas en situación de dependencia en España (2005), la dependencia implica una relación compleja entre las condiciones de salud alteradas, funciones o estructuras corporales afectadas, limitaciones para realizar diversas actividades y los factores del contexto, tanto personal, como ambiental en el que se desenvuelve la persona, lo que implica la necesidad de considerar múltiples parámetros, que habitualmente son valorados mediante diferentes metodologías de medición (que conllevan importantes dificultades o carencias): métodos objetivos de medición, observar 
el funcionamiento en la realización de diversas actividades y medición de las ayudas en lugar de las actividades.

La valoración de la dependencia consiste fundamentalmente en el estudio de ciertas actividades en las cuales la persona dependiente necesita de la ayuda de otra persona y de la intensidad de dicha ayuda, sin olvidar por supuesto su interacción con el contexto ambiental y personal, ni con las deficiencias. A pesar del reconocimiento general de lo anterior en la actualidad no se cuenta con un método o instrumento unificado y consensuado internacionalmente, si no que se utilizan múltiples criterios para definir y clasificar la dependencia, así como diferentes baremos y escalas, que en su mayoría no hacen referencia explícita a la dependencia (sino que utilizan términos como incapacidad física, funcional, funcionamiento físico y problemas estructurales, actividades de la vida diaria), lo que refleja que no sólo existen problemas para contar con métodos de valoración de la dependencia, sino también una importante confusión de términos. Existen por tanto multitud de escalas que se utilizan para la evaluación de la dependencia 0 aspectos relacionados, por citar algunas: Indice de actividades de la vida diaria de Katz (ADL, Katz, Ford y Moskowitz, 1963), Índice de Barthel (Mahoney y Barthel, 1965), Escala de Habilidades Adaptativas (ABS, AAMR, 2002), etc.

Estas diferentes escalas de dependencia tienen diferentes usos (lo que conlleva diferencias importantes en cuanto a sus características): calcular el gasto que generan, evaluación y control del personal, seguimiento y planificación de los cuidados prestados, controles de calidad y gestión del gasto que generan; adjudicación de plazas, realización de estudios estadísticos y sociológicos de salud y calidad de vida.
Además, las escalas también difieren en cuanto a las fuentes de información utilizadas en función del momento o lugar de la valoración: profesionales de la salud (médicos o personal de enfermería), profesionales sociales (trabajadores sociales, cuidadores, etc.) y el propio interesando o sus familiares, así como los métodos de obtención de información también varían de forma importante (Observación directa, entrevista al interesado, familiares, cuidadores, formularios de autovaloración)

\section{Avances en el acuerdo internacional sobre la evaluación de la discapacidad}

\section{La Clasificación internacional del funcionamiento de la discapacidad $y$ de la salud (CIF)}

En un intento de unificar y simplificar terminología y sistemas de graduación de los problemas relacionados con discapacidad la OMS ha desarrollado la "Clasificación internacional del funcionamiento de la discapacidad y de la salud (CIF)", una herramienta útil y precisa, reconocida a nivel internacional, que ayuda al diagnóstico, valoración, planificación e investigación del funcionamiento y la discapacidad, diseñada para ser utilizada en varias disciplinas y diferentes sectores.

Concretamente los objetivos de la CIF son los siguientes:

- Proporcionar una base científica para la comprensión y el estudio de la salud y los estados relacionados con ella, los resultados y los determinantes.

- Establecer un lenguaje común para describir la salud y los estados relacionados con ella, para mejorar la comunicación entre distintos usua- 
rios, tales como profesionales de la salud, investigadores, diseñadores de politicas sanitarias y la población general, incluyendo a las personas discapacitadas.

- Permitir la comparación de datos entre países, entre disciplinas sanitarias, entre los servicios y en diferentes momentos a lo largo del tiempo.

- Proporcionar un esquema de codificación sistematizado para ser aplicado en los sistemas de información sanitaria.

La CIF entiende la discapacidad como "una interacción multidirección entre la persona y su contexto socio-ambiental", lo que da lugar a un sistema de clasificaciones y sub-clasificaciones, así como un glosario de términos, definiciones, vocabularios, etc. No se refiere únicamente a las limitaciones para realizar actividades, sino a la interacción de todos los componentes. Esta clasificación considera diferentes factores a la hora de realizar la valoración de la dependencia. Concretamente se consideran dos grandes bloques: Funcionamiento y discapacidad que incluye información sobre Funciones y Estructuras corporales y sobre Actividades y Participación) y Factores contextuales (Factores ambientales y Factores personales).

\section{CLASIFICACIÓN INTERNACIONAL DEL FUNCIONAMIENTO, DE LA DISCAPACIDAD Y DE LA SALUD (CIF, OMS, 2001)}

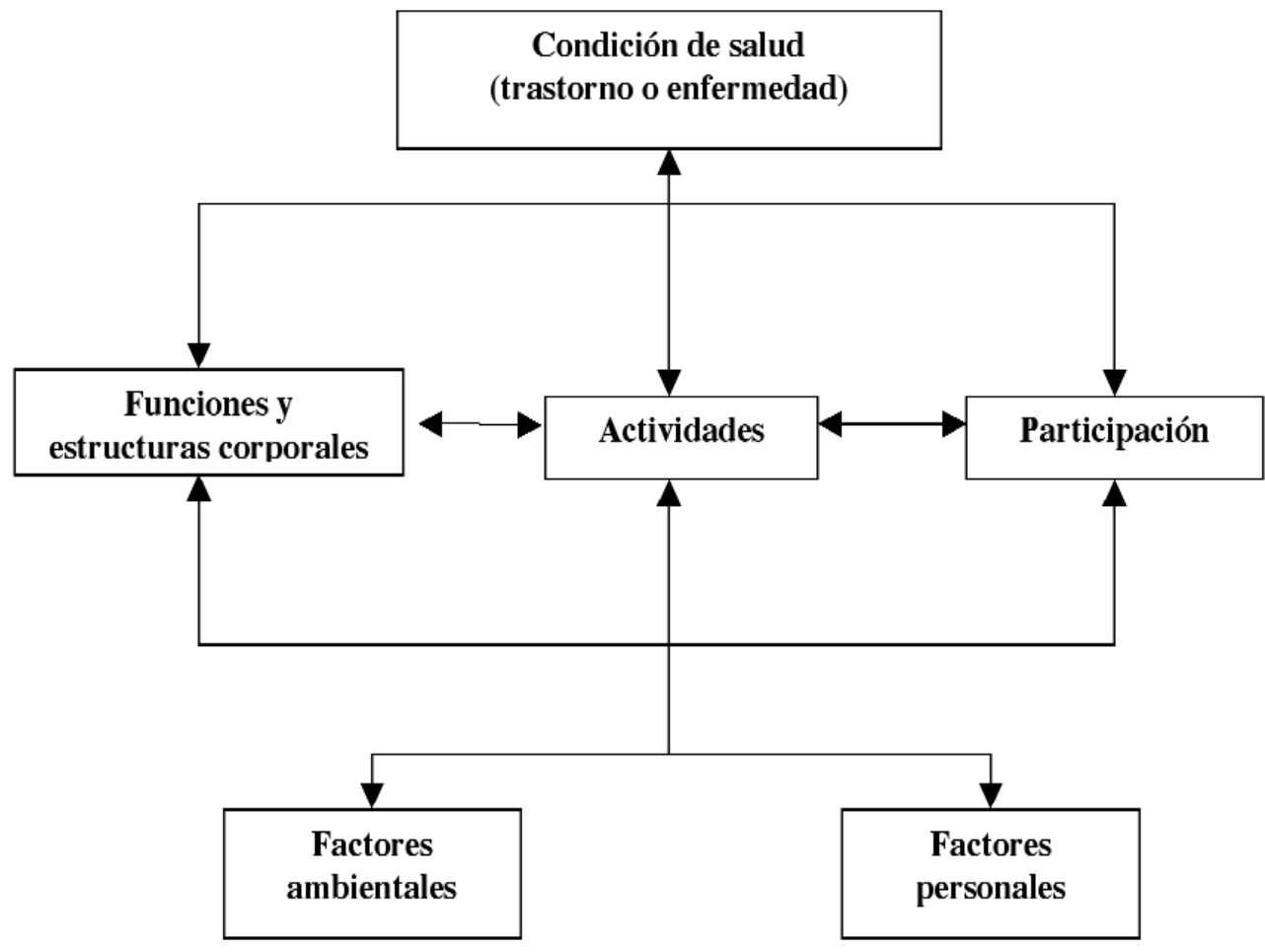


Las características del CIF hacen que sus aplicaciones sean muy diversas. Esta herramienta puede usarse:

- Como herramienta estadística (ej. Encuestas o estudios de población).

- Como herramienta de investigación, para medir resultados, calidad de vida o factores ambientales.

- Como herramienta clínica, en la valoración de necesidades, para homogeneizar tratamientos con condiciones específicas de salud, en la valoración vocacional, en la rehabilitación y en la evaluación de resultados.

- Como herramienta de política social, en la planificación de sistemas de seguridad social, sistemas de compensación y para diseñar e implementar políticas.

- Como herramienta educativa, para el diseño del currículum y para aumentar la toma de decisiones de conciencia de la sociedad y para poner en marcha actividades sociales.

- La CIF se emplea en sectores como las compañías de seguros, la seguridad social, el sistema laboral, la educación, la economía, la política social, el desarrollo legislativo y las modificaciones ambientales.

\section{Áreas consideradas en la evaluación de la dependencia}

Como se señala en el Libro blanco de la dependencia, a pesar de la gran variedad de escalas y métodos de evaluación de la dependencia existentes y sus diferentes características, los métodos utilizados para la medición de la dependencia habi- tualmente se basan en la capacidad de las personas para realizar diversas actividades cotidianas, concretamente en la necesidad de ser ayudado en la realización de actividades de la vida diaria, dentro de las cuales se incluyen diferentes actividades sobre las cuales tampoco hay consenso. A pesar de esa falta de contexto, parece que las áreas más frecuentemente incluidas son: la necesidad de ayudas técnicas para movilidad, las actividades de autocuidado (higiene de excreción, comer, beber, lavarse, vestirse, cuidado de las partes del cuerpo) y de los de movilidad (andar y cambiar las posturas del cuerpo).

Estas diferencias (y acuerdo en las áreas señaladas) se hacen patentes también tras observar los aspectos considerados entre los diferentes países, como puede verse en la siguiente tabla.

La Asociación Americana de Retraso Mental, intentando avanzar en esa línea, propone algunas áreas, concretamente: comunicación, cuidado personal, vida de hogar, HHSS, utilización de la comunidad, autogobierno, salud y seguridad, habilidades académicas funcionales, ocio y trabajo.

La CIF también supone un avance en este sentido; se incluyen diferentes áreas identificadas, definidas, clasificadas y consensuadas internacionalmente en 9 grupos de actividades.

- Aprendizaje y aplicación del conocimiento.

- Tareas y demandas generales.

- Comunicación.

- Movilidad.

- Autocuidado.

- Vida doméstica.

- Interacciones y relaciones personales.

- Áreas principales de la vida Educación/Trabajo/Economía

- Vida comunitaria, social y cívica. 
Tabla 1. Áreas de evaluación de dependencia en diferentes países

\begin{tabular}{|c|c|c|}
\hline $\mathrm{l} R \Lambda \mathrm{NCI} \Lambda$ & $\mathrm{US} \Lambda$ & $\Lambda \mathrm{LLM} \Lambda \mathrm{NI} \Lambda$ \\
\hline I Iigiene & Iavarse & $\begin{array}{ll}\text { I Iigiene personal (incluye } \\
\text { control de csfínteres) }\end{array}$ \\
\hline Vestirse & Vestirsc & Movilidad (incluyc vestirsc) \\
\hline Alimentación & Comer & Alimentación \\
\hline Transferencias & Transferencia cama-sillón & Tarcas domćsticas \\
\hline Control de csfínteres & Control de csfínteres & \\
\hline $\begin{array}{l}\text { Desplazamicntos en cl } \\
\text { interior }\end{array}$ & Ir al lavabo y utilizarlo & \\
\hline \multicolumn{3}{|l|}{ Orientación } \\
\hline Coherencia & & \\
\hline
\end{tabular}

\section{Situación actual española}

\section{Proyecto de Ley Promoción de la autonomía personal y atención a las personas en situación de dependencia}

Actualmente en nuestro país se está trabajando en el Proyecto de Ley Promoción de la autonomía personal y atención a las personas en situación de dependencia (5 de mayo de 2006), que pretende atender las necesidades de la población en situación de dependencia en España, entendiendo como dependencia el estado de carácter permanente en que se encuentran las personas que, por razones derivadas de la edad, la enfermedad o la discapacidad, y ligadas a la falta o a la pérdida de autonomía fisica, mental, intelectual o sensorial, precisan de la atención de otra u otras personas o ayudas importantes para realizar actividades básicas de la vida diaria.
Esta Ley tiene como objetivo fundamentalmente regular las condiciones básicas que garanticen el derecho a la promoción de la autonomía personal y atención a las personas en situación de dependencia, mediante la creación de un Sistema Nacional de Dependencia, que contemplará medidas en todas las áreas que afectan a estas personas. Este Sistema Nacional de Dependencia responderá a una acción coordinada entre las Administración General del Estado y las Comunidades Autónomas, y en su caso Entidades Locales.

En este Proyecto de ley se definen diferentes conceptos relacionados con la dependencia como son: autonomía, dependencia, cuidados familiares, cuidados profesionales, tercer sector y actividades básicas de la vida diaria y se aborda también el tema de la valoraciốn de la dependencia. En primer lugar, se definen diferentes grados de dependencia, de la forma siguiente:

\begin{tabular}{|c|c|}
\hline Grado I & $\begin{array}{l}\text { Dependencia moderada: cuando la persona necesila ayuda para realizar } \\
\text { varias actividades básicas de la vida diaria, al menos una vc } \angle \text { al día. }\end{array}$ \\
\hline Grado II & $\begin{array}{l}\text { Dependencia scvera: cuando la pcrsona nocesita ayuda para rcalizar varias } \\
\text { actividades básicas de la vida diaria dos o tres veces al día, pero no requiere } \\
\text { la presencia permanente de un cuidador. }\end{array}$ \\
\hline Grado III & $\begin{array}{l}\text { Dependencia severa: cuando la persona necesita ayuda para realizar varias } \\
\text { actividades básicas de la vida diaria varias veces al día y, por su pérdida } \\
\text { lotal de autonomía física, mental, intelectual o scnsorial necesila la } \\
\text { prescncia indispensable y continua de la persona. }\end{array}$ \\
\hline
\end{tabular}


Cada uno de estos grados de dependencia se clasificará en dos niveles, en función de la autonomía de las personas y de la intensidad del cuidado que requiere.

Respecto a la valoración de la dependencia, también se aportan algunas directrices generales. El Proyecto de Ley establece que las Comunidades Autónomas determinarán los órganos de valoración de la situación de dependencia, que emitirán un dictamen sobre el grado y nivel de dependencia, con especificación de los cuidados que la persona pueda requerir. También se recoge que se establecerá un baremo para determinar el grado y niveles de dependencia, que tendrá entre sus referentes la CIF, y que establecerá los criterios objetivos de valoración del grado de autonomía de la persona, de su capacidad para realizar las distintas actividades de la vida diaria, los intervalos de puntuación de cada uno de los grados y niveles de dependencia, así como el protocolo con los procedimientos y técnicas a seguir para la valoración de las aptitudes observadas, en su caso. La valoración se realizará teniendo en cuenta las ayudas técnicas, órtesis y prótesis prescritas y el entorno en el que vive la persona en situación de dependencia.

\section{Un ejemplo práctico de evaluación de resultados de programas dirigidos a enfermos mentales graves y crónicos}

Hasta este momento se ha abordado la importancia de la evaluación a la hora de determinar la existencia y el nivel de dependencia en una determinada persona. Sin embargo, la evaluación puede ir un paso más allá, abordando los resultados de las intervenciones. Las personas en situación de dependencia presentan necesidades y demandas concretas. En estos colectivos se acumulan múltiples carencias de muy distinto tipo y se han desarrollado, y como hemos visto se van desarrollar en el futuro, diferentes acciones para intentar responder a esas demandas. La evaluación o valoración de programas se convierte en un recurso social básico para contrastar si esas necesidades y demandas existentes se han resuelto mediante la aplicación de los programas diseñados y aplicados al efecto. De hecho, en opinión de algunos autores (Cohen y Franco, 1993) no hay posibilidad de llevar a la práctica politicas sociales eficaces y eficientes si no se evalúa su aplicación. Con este objetivo se desarrolla la valoración de programas, que podría ser definida como un juicio de valor sistemático realizado mediante procedimientos del método científico, que proporciona de manera válida y fiable, información pertinente que nos permite tomar decisiones concretas (Aguilar y Ander-Egg, 1992).

Esta definición subraya la finalidad práctica de la evaluación: proporcionar informaciones que ayuden a la toma de decisiones racionales e inteligentes en relación con la marcha de los programas. Una de las informaciones más relevantes hace referencia a los resultados obtenidos por dichos programas.

Existen diferentes colectivos dentro de las personas en situación de dependencia: fundamentalmente mayores, pero también otros como son las personas con enfermedad mental grave y crónica. Estas personas están en un riesgo real de llegar a situaciones de dependencia. A continuación nos gustaría exponer una experiencia concreta llevada a cabo en nuestro contexto sobre la evaluación de resultados de programas dirigidos a personas con enfermedad mental grave y crónica.

En la Comunidad de Madrid, la Consejería de Familia y Asuntos Sociales ha 
desarrollado una red de recursos de atención a personas con enfermedad mental grave y crónica alrededor de los Centros de Rehabilitación Psicosocial en los que se ha venido desarrollando un estilo de atención fundamentado en la Rehabilitación Psicosocial como eje director de la intervención, buscando el mantenimiento de estas personas en la comunidad en las mayores condiciones posibles de autonomía. Estos recursos comenzaron a desarrollarse a finales de los años $80^{\circ}$ aunque ha tenido un desarrollo muy importante a partir de la probación en el año 2003 del Plan de atención social a personas con enfermedad mental grave y crónica 2003-2007, uno de cuyos principales objetivos (aunque no el único) es aumentar la red de recursos disponibles y la organización y planificación de la atención a este colectivo y sus familias. En la figura siguiente quedan representados los recursos que conforman esta red.

El Plan recoge un marcado interés por la evaluación, tanto global como de los recursos y acciones que aparecen contempladas dentro del mismo, y la reconoce como un elemento fundamental para la dirección y organización del Plan. Esta evaluación ha sido desarrollada desde el primer momento por un equipo de trabajo de la Universidad Complutense de Madrid dirigido por el Dr. Manuel Muñoz, siempre en estrecha colaboración con los responsables del Plan y los profesionales de los diferentes recursos.

Este trabajo de evaluación se ha lleva-

Figura 1. Recursos de atención social a personas con enfermedad mental grave y crónica, de la Comunidad de Madrid
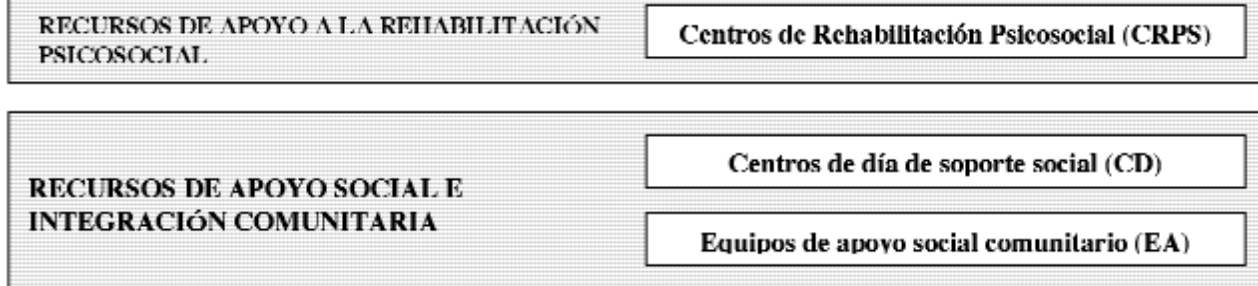

RECURSOS DE REHABILITACIÓN Centros de Rehabilitación Laboral (CRI)

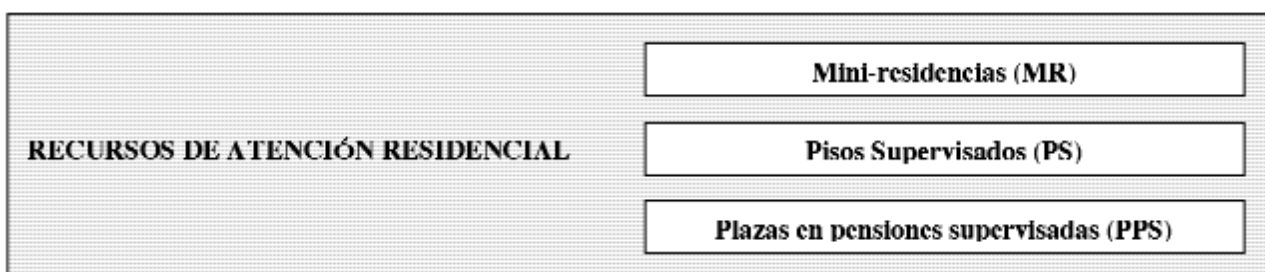

RECURSOS DE ATENCIÓN PARA PERSONAS CON TRASTORNOS MENTALES SIN HOGAR

PRISEMI 
do a cabo en diferentes fases y momentos y con diferentes objetivos. Así se ha realizado a lo largo de estos años la evaluación de la evaluabilidad del Plan, de la implementación, del proceso y funcionamiento así como de los resultados obtenidos. En este caso nos centraremos en la evaluación de los resultados de los diferentes tipos de centros.

En primer lugar, teniendo en cuenta las características de la población objetivo de los recursos que se incluyen en el Plan se identificaron indicadores comunes para evaluar los resultados de los distintos recursos, por ejemplo, satisfacción, tasa de abandono y de altas, sintomatología psiquiátrica, dias de hospitalización psiquiátrica, calidad de vida, funcionamiento psicosocial y bienestar general. A estos indicadores se añadieron algunos específicos para cada tipo de recursos, en función de sus objetivos concretos y sus peculiaridades.

En algunos recursos estos indicadores son suficientes para conocer los principales resultados obtenidos por los cen- tros. Esto es especialmente evidente en algunos tipos de centros como los Centros de Rehabilitación Laboral. Estos centros tienen como misión preparar a las personas afectadas de enfermedades mentales crónicas que tienen dificultades específicas para su acceso al mundo laboral para su reinserción laboral y apoyarles en la búsqueda y mantenimiento de un puesto de trabajo en el mercado laboral. La tabla siguiente, incluye los principales indicadores considerados en las memorias anuales de los centros que hacen referencia a los resultados.

Sin embargo en otros recursos estos indicadores son menos claros o más escasos, debido a que sus objetivos hacen referencia a aspectos menos concretos, por ejemplo, desarrollar autonomia personal y social, apoyar procesos globales de integración social $\mathrm{u}$ ofrecer soporte social. Por ello, fue necesario plantearse la necesidad de introducir algunas medidas que aportaran información complementaria sobre indicadores de resultados, para lo cual se decidió uti-

Tabla 2. Indicadores de resultados incluidos en las memorias de los CRL

\begin{tabular}{|c|}
\hline liormación \\
\hline $\mathrm{N}^{\circ}$ de personas que acceden a formación ocupacional \\
\hline $\mathrm{N}^{\circ}$ de personas que acceden a formación reglada \\
\hline Empleo \\
\hline $\mathrm{N}^{\circ}$ de usuarios con actividad laboral durantc cl año \\
\hline $\mathrm{N}^{\circ} \mathrm{de}$ integraciones laborales \\
\hline $\mathrm{N}^{\circ} \mathrm{de}$ cmplcos por tipo de cmpresa: cmpresa ordinaria, protcgida, marginal \\
\hline $\mathrm{N}^{\circ} \mathrm{de}$ cmplcos en cmpresas privadas y en cl ámbito público \\
\hline $\mathrm{N}^{\circ}$ de cmplcos por tipo de contrato \\
\hline $\mathrm{N}^{\circ}$ de cmplcos sin contrato \\
\hline $\mathrm{N}^{\circ} \mathrm{dc}$ cmplco por tipo de jornada \\
\hline $\mathrm{N}^{\circ}$ de cmplcos dentro del Plan \\
\hline $\mathrm{N}^{\circ}$ de usuarios que continúan cmplcados a final de año \\
\hline $\mathrm{N}^{\circ}$ de personas que han finalizado algún empleo \\
\hline $\mathrm{N}^{\circ}$ de empleos finalizados \\
\hline Motivos para la finalización de los empleos \\
\hline Duración de los empleos finalizados \\
\hline
\end{tabular}


lizar una batería de instrumentos de evaluación aplicados por los propios profesionales de los centros.

Se valoró la posibilidad de hacer un estudio de investigación específico sobre los resultados obtenidos por estos centros, lo que aportaria una mayor calidad y garantía científica pero se descartó porque se pensó que sería más interesante que no fuese algo limitado en el tiempo y realizado exclusivamente de forma externa, si no que el interés por los resultados se debía instalar en la dinámica de los propios centros, aunque tuviese que ser un trabajo apoyado y supervisado de forma externa, por el equipo evaluador, para que la carga de trabajo sobre los profesionales no fuese excesiva.

El proceso para el incremento de la información sobre los resultados de los diferentes recursos fue el siguiente:

1. En primer lugar, se llevó a cabo una exhaustiva revisión de los dominios más utilizados en los trabajos de evaluación de este tipo de recursos. Tras la revisión exhaustiva de los dominios más considerados en este tipo de evaluaciones, se seleccionaron los que se pensaron que eran más relevantes en este tipo de recursos, en relación con los objetivos de los mismos. Los dominios propuestos fueron: Calidad de vida y funcionamiento psicosocial, además de mantener la evaluación de la satisfacción de los usuarios con el recurso (que ya era evaluada en algunos tipos de recursos), aunque adaptándolo a las necesidades de cada recurso.

El interés de la Calidad de vida de las personas con enfermedad mental crónica ha sido una constante en el desarrollo de los programas de atención a este colectivo y, de hecho, su mejora supone uno de los objetivos explicitos de estos programas. Por tanto, la calidad de vida es una variable relevante en la valoración de programas de intervención psicosocial, en general, y en los dirigidos a la rehabilitación de los enfermos mentales crónicos en particular. Si los programas de intervención persiguen aumentar la calidad de vida de los enfermos mentales crónicos, será fundamental contar con medidas que informen sobre los logros conseguidos en este sentido. De hecho algunos autores han llegado a identificar la calidad de vida como la variable más importante para evaluar los resultados de los servicios comunitarios en pacientes crónicos (Tessler y Goldman, 1982). La medida global de calidad de vida puede ser muy adecuada como medida ya que es un marcador indirecto del estado de salud global, y de adaptación social de la persona, y por lo tanto del impacto de la intervención.

El funcionamiento psicosocial también supone uno de los aspectos más relevantes a la hora de analizar los logros de las intervenciones de este tipo. De hecho, es una de las variables más usadas para medir la eficacia de los tratamientos, junto a sintomas, salud y bienestar subjetivos (Muñoz et al, 1999). Los programas de rehabilitación psicosocial se constituyen en el tratamiento, bajo cuyos efectos los individuos debieran adquirir una serie de habilidades que les doten de una mayor autonomía funcional, lo que supuestamente implicaria un mayor bienestar global.

En cuanto a la satisfacción de los usuarios, resulta paradójico el hecho de que en la valoración de programas de intervención social se hayan considerado las necesidades, recursos, costes y beneficios de los servicios prestados y sin embargo, el punto de vista del usuario 
haya sido frecuentemente ignorado. Tal vez podría explicarse este fenómeno si se atiende a las dificultades para interpretar los niveles de satisfacción, a la falta de una base significativa para establecer comparaciones 0 a los problemas existentes para obtener una muestra representativa de usuarios. La satisfacción puede ser utilizada como una medida de la efectividad de una intervención. Así un nivel global de satisfacción puede servir como indicador de resultado para comparar diferentes programas de intervención e igualmente puede emplearse para comparar el mismo programa bajo diferentes condiciones, aunque, por supuesto no deba considerarse como el único, ni el mejor, indicador de la efectividad. De hecho, se ha encontrado que la satisfacción se relaciona sistemáticamente con otras medidas del éxito del tratamiento, aunque la correlación no es perfecta, lo que indica que se trata de una medida de resultado importante, pero no la única que define el éxito: no puede reemplazar a otros indicadores de resultado en la evaluación de la calidad de la atención.

2. Tras seleccionar algunos de estos dominios, se buscaron aquellos instrumentos utilizados para su evaluación. Los criterios utilizados para buscar y seleccionar dichos instrumentos fueron principalmente:

- Utilización previa en estudios de evaluación de resultados de programas dirigidos a este colectivo.
- Criterios psicométricos adecuados.

- Brevedad.

- Facilidad de aplicación.

- Versión en castellano.

Teniendo en cuenta dichos criterios, la propuesta de instrumentos de evaluación de los dominios seleccionados fue la siguiente (final de página):

Los dos sistemas de clasificación de trastornos mentales más reconocidos internacionalmente, DSM-IV-TR y CIE10 , recogen dos instrumentos de evaluación de funcionamiento / discapacidad: EEFG (APA, 1994) y WHO DAS-I (1992). Estos son los instrumentos que se propusieron.

La Escala de Evaluación de la Actividad Global (EEAG) (Asociación Psiquiátrica Americana, 1994) es un instrumento dirigido a medir el funcionamiento psicosocial del individuo. Es el instrumento empleado en el eje 5 del DSM-IV para la evaluación de la actividad general. El EEAG es una escala de 100 puntos en la que el profesional debe evaluar tanto la gravedad de los sintomas que presenta la persona como la gravedad del deterioro en la actividad escolar, laboral o social. La evaluación final viene determinada por la peor de las dos evaluaciones.

El WHO-DAS I es el instrumento recomendado por la OMS (CIE-10) para la

\begin{tabular}{|l|l|}
\hline Dominios & Instrumcntos \\
\hline Calidad de vida & $\begin{array}{l}\text { Satisfaction with life Domains Scale - SLDS (Baker e } \\
\text { Intagliata, 1982). }\end{array}$ \\
\hline l'uncionamicnto psicosocial & $\begin{array}{l}\text { Global } \Lambda \text { ssessment of liunctioning - L LI'G ( } \Lambda \text { sociación } \\
\text { Psiquiátrica Americana, 1994). } \\
\text { WHO Short Disability } \Lambda \text { sscssment Schcdulc - WHO D } \Lambda \text { S-I } \\
\text { (Organización Mundial de la Salud, 1992) }\end{array}$ \\
\hline Satisfacción de los usuarios & Cuestionario de Satisfacción Global (Lópè, 1992) \\
\hline
\end{tabular}


evaluación de las dificultades provocadas por la presencia de problemas mentales o físicos. Proporciona una guía de las áreas que el profesional debe evaluar durante el transcurso de la entrevista que son: cuidado personal, ocupación, familia/vivienda y funcionamiento social (relación con los miembros de la comunidad y participación en actividades sociales). En función de la información obtenida, el profesional debe puntuar el deterioro de la persona en una escala analógico visual de 0 ("ausencia de deterioro") a 5 ("gran deterioro") de acuerdo a unos criterios definidos. Además de evaluar el deterioro en las áreas específicas de funcionamiento, el entrevistador debe decidir el período de tiempo cubierto en la evaluación ("actual", "último mes" u "otro"), así como especificar la duración total del deterioro ("menos de un año", "un año más" o "desconocido") y los recursos específicos que presenta la persona.

Tras la propuesta de instrumentos aparecieron algunos problemas con el SLDS para la evaluación de la calidad de vida. Sin embargo no se encontró otro instrumento que se ajustase mejor a las demandas de los profesionales por lo que se decidió realizar un nuevo instrumento de evaluación partiendo de éste y respetando su esquema de formulación de preguntas y su escala de respuestas. Por lo tanto sobre el SLDS se realizaron diferentes modificaciones a partir de la opinión de los profesionales de los centros.

3. Después se llevó elaboró una propuesta de dominios e instrumentos que fue presentada y discutida con los responsables del Plan y los directores de los centros. De forma simultánea se presentaron a los responsables del Plan los resultados principales de la búsqueda de dominios e instrumentos.
4. Tras esto se realizaron algunas modificaciones y se elaboró un borrador.

5. Este borrador fue aplicado en un estudio piloto que fue llevado a cabo en recursos de diferente tipo para identificar posibles problemas en su aplicación.

6. Los resultados del estudio piloto indicaron la necesidad de realizar algunos pequeños ajustes en los instrumentos.

7. A continuación se determinó el procedimiento para aplicación de dichos instrumentos. Concretamente se acordó que estos instrumentos se aplicarían de la siguiente manera:

- Cuestionario de calidad de vida e instrumentos de evaluación del funcionamiento: Se aplicarán en el momento de incorporación de la persona al centro, en el momento de la finalización de la intervención y a final de año a todos aquellos usuarios que se encuentren en atención en el centro.

- Cuestionario de satisfacción de los usuarios: Se aplicará en el momento en que el usuario finalice su intervención en el centro $\mathrm{y}$ de forma sistemática a final de año a todos aquellos usuarios que se encuentren en atención en el centro.

8. Se distribuyó la versión definitiva de los instrumentos y del procedimiento de aplicación a todos aquellos centros, así como las bases de datos diseñadas para la codificación de la información de los instrumentos. 
9. Tras este proceso comenzó la aplicación sistemática en los centros a finales de 2005.

Tras comenzar la aplicación masiva en los centros aparecieron algunos pequeños problemas, que se solucionaron mediante la elaboración de una pequeña guía con algunas indicaciones sobre la aplicación de estos instrumentos y algunos ejemplos.
Hasta el momento sólo se disponen de los datos de los usuarios en atención a finales de 2005, por lo que todavía no se dispone de datos sobre el cambio de los usuarios a través de los diferentes momentos de evaluación en las diferentes variables consideradas. Sin embargo los datos analizados hasta el momento indican la utilidad de los instrumentos utilizados para detectar diferencias en los perfiles de los usuarios atendidos por los diferentes centros. En breve se dispondrá de más datos.

\section{BIBLIOGRAFÍA}

Aguilar, M.J. y Ander-Egg, E. (1992). Evaluación de servicios y programas sociales. Madrid: Siglo XXI.

American Psychiatric Association (2000). DSMIV-TR. Washington: Psychiatric Press

Baker, F. e Intagliata, J. (1982). Quality of life in the evaluation of community support systems. Evaluation and program planning, 5, 69-79.

Cohen, E. y Franco, R. (1993). Evaluación de Proyectos sociales. México: Siglo XXI. Global.
Ministerio de Trabajo y Asuntos Sociales (2005). Libro Blanco sobre la "atención a las personas en situación de dependencia en España. Madrid.

Muñoz, M., Vázquez, C., Muñiz, E., LópezLuengo, B., Hernangómez, L y Díaz, M. (1999). Evaluación de resultados en la esquizofrenia: el ejemplo de la comunidad autónoma de Madrid. Intervención psicosocial, 8, 1, 73-87.

Querejeta (2003). Discapacidad / Dependencia. Unificación de criterios de valoración y clasificación. Guipuzkoa.

World Health Organization (1996). CIE-10: Multiaxial version. Geneve: WHO. 